\title{
Nachlässe von Germanistinnen und Germanisten aus der DDR: eine Beständeübersicht
}

\author{
Simone Waidmann / Frederike Teweleit / Ruth Doersing
}

Die nachfolgende Beständeübersicht ist als heuristisches Arbeitsinstrument zu verstehen, das keinerlei Anspruch auf Vollständigkeit erhebt. Sie beruht auf Recherchen in öffentlich zugänglichen Nachweisinstrumenten und Selbstauskünften bestandshaltender Institutionen.

Neben Literaturwissenschaftlerinnen und Literaturwissenschaftlern wurden in Auswahl auch germanistische Linguistinnen und Linguisten berücksichtigt. Aufnahme in die Übersicht fanden nur Bestände (Nachlässe und Vorlässe), die von den genannten Personen bzw. deren Erben gebildet wurden. Instituts- und Gremienunterlagen, Promotions- und Habilitationsakten, Personalakten von Arbeitgebern und andere durch Dritte gebildete Bestände, u. a. Stasiakten, bleiben unberücksichtigt. Die Heterogenität der Angaben ist auf die sehr unterschiedlichen Erschließungsstände in den jeweiligen Archiven zurückzuführen.

\section{Becker, Henrik (1902-1984)}

\section{Universitätsarchiv Jena}

Nachlass (5,25 lfm, erschlossen, Findbuch)

Inhalt: Lehrtätigkeit, hier Unterlagen über die Tätigkeit an der Volkshochschule und der $\mathrm{ABF}$ in Leipzig sowie am Germanistischen Institut und dem Institut für Sprachpflege und Wortforschung der FSU Jena. Mitarbeit in Arbeitsgemeinschaften und Kommissionen, hauptsächlich Sprachlehrbücher des Sprachlehrbuchausschusses der Gewerkschaft der Lehrer und Erzieher im FDGB Leipzig, auch Unterlagen aus der Tätigkeit in Fachkommissionen des Deutschen Pädagogischen Zentralinstituts Berlin und der Arbeitsgemeinschaften der Abteilung Sprachunterricht der TH Dresden und der Arbeitsgemeinschaft Technische Begriffe der Hochschule für Elektrotechnik Ilmenau. Unveröffentlichte Manuskripte/Typoskripte linguistischer und philologischer Arbeiten und Korrespondenz dazu sowie literaturgeschichtlicher und literaturtheoretischer Arbeiten, Übersetzungen, Rezensionen und Gutachten, Magnettonbänder, Sonderdrucke und Zeitschriftenartikel, Rezensionen zu Arbeiten von H. Becker. Manuskripte anderer Autoren, Sonderdrucke von anderen Autoren, persönliche Unterlagen, u. a. autobiographische Aufzeichnungen, Briefwechsel.

\section{Bierwisch, Manfred (*1930)}

Archiv der Berlin-Brandenburgischen Akademie der Wissenschaften 
Vorlass (1 lfm, nicht erschlossen)

Inhalt: $k$.A.

\section{Bischoff, Karl (1905-1983)}

Karl-Bischoff-Archiv, Martin-Luther-Universität Halle-Wittenberg Nachlass (66 lfm, grob erschlossen, Liste)

Inhalt: Sammlungen zum Wortschatz des Mittelelbegebietes: Zettelarchiv (ca. 250.000 Zettel mit ca. 1 Mio. Belegen), 11 von Bischoff versendete Fragebögen 1936-1956, weitere Fragebögen anderer Institutionen, Karten zur Wortgeografie, private Wortschatzsammlungen, Wörterbuchmanuskript, Sammlung von Flurnamen des Mittelelbegebietes, Sammlung von Fotografien zu Sachvolkskunde und Brauchtum, CDs mit Mundartproben, Manuskripte, Aufzeichnungen, Sammlungen von Bischoff, wissenschaftliche Nachlassbibliothek, Materialien zur Biographie von Karl Bischoff.

\section{Boeckh, Joachim Georg (1899-1968)}

Archiv der Berlin-Brandenburgischen A kademie der Wissenschaften

Teilnachlass (7,2 lfm, erschlossen, Findbuch)

Inhalt: Manuskripte und Druckschriften zur Literaturwissenschaft, Jugendbewegung und Politik, Materialsammlungen zu Forschungsthemen und zur Familiengeschichte.

\section{Bundesarchiv Berlin}

Teilnachlass (1 lfm, nicht erschlossen)

Inhalt: Materialsammlung über die Jugendbewegung v. a. der zwanziger Jahre: Rundschreiben, Flugblätter, Mitteilungsblätter, Veröffentlichungen von und über Jugendverbände.

\section{Bunge, Hans (1919-1990)}

Archiv der Akademie der Künste Berlin

Nachlass (16,5 lfm, erschlossen, eigene Datenbank)

Inhalt: Werke, diverse Arbeitsunterlagen, Druckschriften, Korrespondenz, biographische Unterlagen, Erinnerungsstücke, Dokumentation, fremde Werke und Bearbeitungen für die Bühne.

Dietze, Walter (1926-1987)

Universitätsarchiv Leipzig

Nachlass (12 lfm, erschlossen, eigene Datenbank)

Inhalt: Fotos, persönliche Unterlagen, Würdigungen, Materialsammlung, Rezensionen zu Dietzes Werken, Korrespondenz.

Düwel, Hans (1891-1973)

Universitätsarchiv Rostock

Nachlass $(0,5 \mathrm{lfm}$, nicht erschlossen) 
Inhalt: Regionalgeschichtliche und sprachwissenschaftliche Forschungsunterlagen und Materialsammlung zum Dorf Vogtshagen, Skizzen.

Erben, Johannes $(* 1925)$

Archiv der Berlin-Brandenburgischen A kademie der Wissenschaften Vorlass $(1,8 \mathrm{lfm}$, nicht erschlossen $)$

Inhalt: $\mathrm{k}$.A.

Frings, Theodor (1886-1968)

Archiv der Berlin-Brandenburgischen Akademie der Wissenschaften

Nachlass (23,8 lfm, erschlossen, Findbuch)

Inhalt: Persönliche Unterlagen (u. a. Urkunden und Fotografien), Vorlesungsnachschriften, Manuskripte zur Sprach- und Literaturgeschichte, wissenschaftsorganisatorische Unterlagen, Korrespondenz.

\section{Geerdts, Hans Jürgen (1922-1989)}

\section{Universitätsarchiv Greifswald}

Nachlass (2,7 lfm, teilverzeichnet, eigene Datenbank/Liste)

Inhalt: Manuskripte literarischer Werke, Unterlagen zur politischen und gesellschaftlichen Aktivität, zur Lehr- und Verwaltungstätigkeit für die Universität, Biographisches, Korrespondenz und Zeitungsausschnitte.

\section{Germann, Dietrich (1926-2014)}

\section{Universitätsarchiv Jena}

Nachlass (5 lfm, grob erschlossen, Liste)

Inhalt: Materialsammlungen $\mathrm{zu}$ historischen, germanistischen und stadtgeschichtlichen Themen, Vorlesungsmitschriften, Schriftwechsel aus mehreren Jahrzehnten, Manuskripte zu verschiedenen Themen, Unterlagen über seine Mitarbeit an der Schiller-Nationalausgabe und der Heine-Säkularausgabe aus den 1950er/1960er Jahren, Goethe-Studien aus den 1970er Jahren, Presseartikel Germanns aus den 1970er bis 1980er Jahren, Dokumente zum Archivwesen, Unterlagen zur Geschichte des Zentralinstituts für Mikrobiologie und experimentelle Therapie (ZIMET).

Gernentz, Hans-Joachim (1918-1997)

Universitätsarchiv Rostock

Nachlass (1,5 lfm, nicht erschlossen)

Inhalt: Persönliche Unterlagen, Vorträge, Vorlesungen, Gutachten, Rezensionen, Schriftgut des Martin-Luther-Komitees der DDR.

Girnus, Wilhelm (1906-1985)

Archiv der Akademie der Künste Berlin

Teilnachlass (5 lfm, 232 Bände, nicht erschlossen) 
Inhalt: Überlieferung aus dem Zeitraum 1932-1984: Werkmanuskripte vor allem aus publizistischer und wissenschaftlicher Tätigkeit, darunter Aufsätze, Kommentare, Vorträge und Vorlesungen einschließlich zugehöriger Notizen, Entwürfe und Materialsammlungen. Manuskript einer Autobiographie, Unterlagen aus der Arbeit am Berliner Rundfunk (1947/48) und in der Redaktion»Sinn und Form« (1964-1981), geschäftlicher Schriftwechsel, Briefe von Ulrich Becher, Friedrich Dieckmann, Hugo Huppert, Briefkopien von Pierre Bertaux, Bertolt Brecht, Louis Fürnberg, Thomas und Katia Mann, Robert Neumann, Claude Prévost, Ludwig Renn, Romain Rolland, Anna Seghers und André Wurmser. Bibliothek: Primärliteratur, Belletristik, Sachgebiete Literatur, Politik, Geschichte, Philosophie und Kulturpolitik, Jahrbücher, Sonderdrucke, Zeitschriften, insbesondere fremdsprachige, Widmungsexemplare, u. a. von Heinrich Ehmsen, Erich Fried, Gisela Kraft, Hans Pischner, Michael Scharang, Robert Weimann und F. C. Weiskopf sowie in- und ausländischen Wissenschaftlern, Dokumentationsmaterial.

\section{Deutsches Rundfunkarchiv Potsdam-Babelsberg}

Teilnachlass $(0,2 \mathrm{lfm}$, erschlossen, Verzeichnis)

Inhalt: Sendemanuskripte politischer Kommentare von Girnus im Berliner Rundfunk.

\section{Greiner, Martin (1904-1959)}

Universitätsarchiv Leipzig

Nachlass (2,4 lfm, nicht erschlossen)

Inhalt: Manuskripte zu Vorlesungen, Rundfunksendungen, Vorträgen u.a.

\section{Haiduk, Manfred (*1929)}

\section{Archiv der Akademie der Künste Berlin}

Teilvorlass (1,2 lfm, erschlossen, eigene Datenbank)

Inhalt: Sammlung Peter Weiss: Materialsammlung zur Biographie und zum Nachlass von Peter Weiss, Materialsammlung zum Werk von Peter Weiss und zu Theaterinszenierungen, Manuskriptdrucke von Theaterstücken, Beiträge zur Peter-Weiss-Forschung, Manuskripte und Korrespondenz von Manfred Haiduk, Fotos.

\section{Hartinger, Walfried (1938-2003) \\ Deutsches Literaturarchiv Marbach}

Nachlass (3,3 lfm, vorgeordnet, eigene Liste)

Inhalt: Prosa: Arbeitsthesen zur Dissertation B, Ausarbeitungen zum Verhältnis von DDR-Literatur und DDR-Literaturwissenschaften, Mitschriften und Seminararbeiten aus dem Studium, Seminarkonzeptionen, Reden und Vorträge. Herausgegebenes: Vom Umgang mit Lyrik der Moderne (zus. mit Peter Geist). Verschiedenes: Gutachten, Lebenslauf und Publikationslisten, Leistungseinschätzungen über Kollegen, Notizen und Materialien zur DDR-Lyrik, zu Thomas 
Bernhard, zu Christoph Hein, zu Erich Loest u. a. Briefe an ihn (z. T. und Christel Hartinger) von: Waltraud Ahrndt, Annemarie Auer, Volker Braun, Reinhard Bernhof, Heinz Czechowski, Rosa Maria Domaschke (Róža Domašcyna), Kurt Drawert, Benedikt Dyrlich, Volker Ebersbach, Wolfgang Eckert, Franz Fühmann, Peter Gosse, Ralph Grüneberger, Kerstin Hensel, Hildegard Jahn-Reinke, Bernd Jentzsch, Erich Köhler, Rainer Kirsch, Norbert Maron, Karl Mickel, Dieter Mucke, Joachim Nowotny, Walter Petri, Gunter Preuß, Helmut und Brigitte Richter, Trude Richter, Frank Ruddigkeit, Ursula Rüschel, Gerti Tetzner, Wolfgang Trampe, Christa und Gerhard Wolf, Ria und Hartmut Zenker u.a. Dokumente: Materialien zum Bezirkskabinett für Kulturarbeit und zum Schriftstellerverband der DDR, politische Unterlagen, Verlagsvertrag. Manuskripte anderer: Parteigruppenberichte u.a. Briefe anderer: Elfriede Brüning, Gabriele Eckart, Christiane Grosz, Ruth Kraft, Friederike Mayröcker.

\section{Helbig, Gerhard (1929-2008)}

\section{Institut für Deutsche Sprache Mannheim}

Nachlass (0,1 lfm, 2.726 Bände, erschlossen, Verzeichnis)

Inhalt: Nachlassbibliothek, Briefe, Postkarten, Lesezeichen (mit und ohne Notizen), Blätter (mit und ohne Notizen), handschriftliche Vorarbeiten und Briefe zur $\gg$ Deutschen Grammatik «.

\section{Kantorowicz, Alfred (1899-1979)}

\section{Archiv Staats- und Universitätsbibliothek Hamburg}

Nachlass (20 lfm, erschlossen, eigene Datenbank)

Inhalt: Korrespondenzen, Manuskripte, biographische Unterlagen, Drucke.

\section{Karg-Gasterstädt, Elisabeth (1886-1964) \\ Universitätsbibliothek Leipzig \\ Nachlass (ca. 0,1 lfm, nicht erschlossen) \\ Inhalt: Briefe}

\section{Kaufmann, Hans (1926-2000) \\ Deutsches Literaturarchiv Marbach}

Nachlass (3,3 lfm, feingeordnet, Bestandsbeschreibung)

Inhalt: Prosa: »Bertolt Brecht - Tragödie, Komödie, episches Theater (Habilitationsschrift), Vorüberlegungen und Entwürfe zur »Geschichte der deutschen Literatur von den Anfängen bis zur Gegenwart «, »Heinrich von Kleist - zum 200. Geburtstag«, »Politisches Gedicht und klassische Dichtung « (Dissertation), »Probleme der dichterischen Darstellung von Helden aus den unteren Volksschichten in den Anfängen der deutschen Klassik « (Staatsexamensarbeit), »Prosa der DDR in den siebziger Jahren «, Texte, Notizen und Exzerpte zu Bertolt Brecht und zu Heinrich Heine, Texte, Notizen und Gutachten zu Volker Braun, Interviews mit verschiedenen Personen, Laudatio für Sarah Kirsch und Gerhard Holz- 
Baumert. Reden und Vorträge zu verschiedenen Themen, u. a. zu Heinrich Böll, Bertolt Brecht, Alfred Döblin, Hans Fallada, Erich Kästner, Heinrich Mann, Robert Musil, Erwin Strittmatter und Christa Wolf. Rezensionen. Vorlesungen: »Bertolt Brecht«, »Deutsche Komödien seit Lessing «, »Liebeslyrik von Goethe bis Brecht «, »Literatur des demokratischen Deutschland «, »Probleme der Literaturtheorie«, »Vormärz«, »1815-1848«, »1848-1870« u. a., z. T. mit dazugehörigen Notizen und Materialien. Herausgegebenes: Konvolut zur historisch-kritischen Ausgabe der Schriften Bertolt Brechts. Verschiedenes: Notizen und Exzerpte zu verschiedenen Themen und Autoren, Gutachten, Konvolut zum HeineFilm (1972), Konvolute mit Materialien für und Protokolle von Germanistenlehrgängen (mit Manuskripten anderer), Notizen aus der FDJ-Studiengruppe Germanistik, Projektvorschlag von H.K. für ein Großprojekt »Literatur aus der DDR und das Problem der Freiheit. Studien über Repression und Emanzipation«, Skizzen und Vorbereitungen zu einem Lyrik-Abend 1973 u. a. Autobiographisches: »Die dritte Niere«. Briefe an ihn: von Volker Braun, Klaus F. Gille, Kurt Hager, Christa Wolf u. a. Briefe von ihm: an Volker Braun, Lotte Fürnberg, Louis Hay, Werner Hecht, Dieter Schiller, Helene Weigel, Christa Wolf u. a. Zugehörige Materialien: Dokumente zur beruflichen Tätigkeit, Auszeichnungen, Konvolut »Dokumente zum Aufbau der Germanistik in der DDR«, Konvolut »GauckBehörde« u.a. Manuskripte anderer: Volker Braun (»Hans Faust«), Renate Drommer und Herwig Kipping, Erhard Hexelschneider und Michael Wegner, Georg Kaufmann, Gerhard Scholz, Christa Wolf u.a. Zum Nachlass gehören: Zeitungsausschnitte, Rezensionen u. a.

\section{Kettmann, Gerhard (1928-2009)}

\section{Karl-Bischoff-Archiv, Martin-Luther-Universität Halle-Wittenberg}

Nachlass (7 lfm, nicht erschlossen)

Inhalt: $k$.A.

\section{Magon, Leopold (1887-1968)}

Archiv der Berlin-Brandenburgischen Akademie der Wissenschaften

Nachlass $(0,15 \mathrm{lfm}$, erschlossen, Findkartei)

Inhalt: Vorlesungsnachschriften (Kopien) u. a. zu Vorlesungen von G. Roethe, M. Dessoir und E. Schmidt.

\section{Mayer, Hans (1907-2001) \\ Historisches Archiv Köln}

Teilnachlass (28 lfm, erschlossen, Findbücher)

Inhalt: Manuskripte, Druckfahnen von Veröffentlichungen, Reden. Manuskripte fremder Autoren, u. a. v. Ernst Bloch, Bertold Brecht, Friedrich Dürrenmatt, Erich Fried, Max Frisch, Ernst Jandl, Thomas Mann, Stefan Schütz, Christa Wolf. Zeitungsartikel von und über Hans Mayer, Interviews mit Hans Mayer. Kritiken zu Veröffentlichungen, zu Vorträgen und Diskussionen. Einladungen, Program- 
me, Plakate, Berichte zu öffentlichen Auftritten von Hans Mayer (1939 ff.). Persönliche Unterlagen zu Familie und Kindheit in Köln, Emigration in die Schweiz, Chefredakteur bei Radio Frankfurt, Professor in Leipzig, Hannover, Tübingen, Geburtstage, Vortragsgelegenheiten. Briefe von u. a. Theodor W. Adorno, Ilse Aichinger, Jean Améry, Günther Anders, Alfred Andersch, Raymond Aron, Ingeborg Bachmann, Lothar Baier, Johannes R. Becher, Jürgen Becker, Samuel Beckett, Hermann Beil, Hans Bender, Thomas Bernhard, Pierre Bertaux, Michael Marschall von Bieberstein, Max Bill, Ernst Bloch, Karola Bloch, Walter Boehlich, Heinrich Böll, François Bondy, Pierre Boulez, Willy Brandt, Volker Braun, Bertolt Brecht, Joseph Breitbach, Margot von Brentano, Arnolt Bronnen, Peter Brückner, Rüdiger Bubner, Carl Jacob Burckhardt, Roger Callois, Elias Canetti, Cesare Cases, Paul Celan, Walter Dirks, Christoph von Dohnányi, Hilde Domin, Dieter Dorn, Heinz Dramsch, Claus Helmut Drese, Kasimir Edschmid, Clemens Eich, Gottfried von Einem, Hans Magnus Enzensberger, Theodor Eschenburg, Lion Feuchtwanger, Hubert Fichte, Dietrich Fischer-Dieskau, Wolfgang Fortner, Elisabeth Freundlich, Hans Frick, Götz Friedrich, Max Frisch, Franz Fühmann, Hans-Georg Gadamer, Hermann Glaser, Albrecht Goes, Günter Grass, Martin Gregor-Dellin, Peter Härtling, Käte Hamburger, Peter von Haselberg, Christoph Hein, Helmut Heißenbüttel, Hans G. Helms, Hans Werner Henze, Stephan Hermlin, Hermann Hesse, Stefan Heym, Wolfgang und Silvia Hildesheimer, Rolf Hochhuth, Gustav René Hocke, Walter Höllerer, Max Horkheimer, Peter Huchel, Hans Henny Jahnn, Ernst Jandl, Walter Jens, Bernd Jentzsch, Uwe Johnson, Erhart Kästner, Erich Kästner, Joachim Kaiser, Lotte Klemperer, Wolfgang Koeppen, Kurt A. Körber, Leo Kofler, Hermann August Korff, Werner Krauss, Karl Krolow, Hans Küng, Günter Kunert, Reiner Kunze, Hartmut Lange, Heinrich Maria Ledig-Rowohlt, Siegfried Lenz, Rolf Liebermann, Franz Liebl, Erich Loest, Vicco von Bülow (Loriot), Georg Lukács, Anna Mahler, Ernst Gottfried Mahrenholz, Erika Mann, Golo Mann, Thomas Mann, Ludwig Marcuse, Friederike Mayröcker, Luis Meana, Peter de Mendelsohn, Robert Minder, Egon Monk, Adolf Muschg, Robert Neumann, Ursula Pasterk, Helmuth Plessner, Monika Plessner, Henry Racamier, Hans Martin Rahner, William E. Rappard, Ludwig Renn, Hans Werner Richter, Luise Rinser, Harry Rowohlt, Günther Rühle, Peter Rühmkorf, Max Rychner, Albert von Schirnding, Heinz Schneekloth, Albrecht Schöne, Percy Ernst Schramm, Ernst Schröder, Wolfram Schütte, Stefan Schütz, Klaus Schultz, Hans Schwab-Felisch, Anna Seghers, Hinrich Seidel, Gerold Späth, Manès Sperber, Hilde Spiel, Georg Steiner, Karin Struck, Hans Syberberg, Marian Szyrocki, Max Tau, Wolfgang Thierse, Horst Tietz, Franz Tumler, Siegfried Unseld, Guntram Vesper, Klaus Völker, Klaus Wagenbach, Gudrun Wagner, Wieland Wagner, Wolfgang Wagner, Martin Walser, Peter Wapnewski, Kadidja Wedekind-Biel, Peter Weiss, Carl Friedrich von Weizsäcker, Christa Wolf, Yan Baoyn, Arnold Zweig. Glückwünsche, Telegramme zu Geburtstagen, Briefe von politischen Institutionen der Bundesrepublik, auch Österreichs, von Ministerien des Bundes und der Länder, von Bun- 
despräsidenten, von Ministerpräsidenten, Oberbürgermeistern zahlreicher Städte, von Staats-, Landes-, Stadttheatern und -opern, von Botschaften ausländischer Staaten, von wissenschaftlichen und künstlerischen Akademien, von deutschen und ausländischen Universitäten, Hochschulen und Instituten, von Gesellschaften (Gesellschaften für Christlich-Jüdische Zusammenarbeit), Rundfunkanstalten, Zeitungen und Verlagen. Ca. 500 Bücher mit eigenhändigen Widmungen von deutschen und ausländischen Autoren, Komponisten und anderen Künstlern. Fotografien zum Werdegang, zu Reisen. Videobänder, Tonkassetten.

Anmerkung: Der Nachlass Hans Mayers war vom Einsturz des Historischen Archivs Köln 2009 betroffen, wurde aber geborgen und kann nach einer Vorbereitungszeit von vier bis sechs Monaten zugänglich gemacht werden.

\section{Universitätsbibliothek Tübingen}

Teilnachlass (1,5 lfm, nicht erschlossen)

Inhalt: Briefe, Sammelstücke.

\section{Mittenzwei, Werner (1927-2014)}

\section{Walter A. Berendsohn Forschungsstelle für deutsche Exilliteratur}

Dienstlicher Teilnachlass $(0,5 \mathrm{lfm}$, erschlossen, Kalliope)

Inhalt: Materialien zu den Büchern »Das Zürcher Schauspielhaus « und »Exil in der Schweiz«, Zeitdokumente (u. a. Programmhefte, Flugblätter, Zeitschriften der Exilanten), Exzerpte, Notizen, Zeitungsartikel, Rezensionen, Aufzeichnungen von Zeitzeugengesprächen mit Sylta Busse, Hermann Scherchen, Wolfgang Heinz, Carl Meffert (Clément Moreau), Fotos von Personen und Aufführungen des Zürcher Schauspielhauses, Audioaufzeichnungen von Zeitzeugengesprächen.

\section{Deutsches Literaturarchiv Marbach}

Nachlass (2,6 lfm, grob geordnet, eigene Liste)

Inhalt: Manuskripte: Entwurf zu einer Literaturtheorie (ausgehend von Brecht und Lukács), Geschichte des Aufbau-Verlags (1945-1992), ausführliche Protokolle zu einer Diskussion mit Bertolt Brecht am Berliner Ensemble über den 17. Juni 1953, mit Peter Hacks zu Regiefragen, Rezensionen (1960-2007). Briefe an und von: Volker Braun, Ernesto Grassi, Klaus Gysi, Peter Hacks, Hans Kaufmann, Kurt Pinthus, Siegfried Unseld, Helene Weigel u. a. Briefe und Protokolle zur dramaturgischen Tätigkeit am Berliner Ensemble.

\section{Müller, Joachim (1906-1986) \\ Universitätsarchiv Jena}

Nachlass ( $9,35 \mathrm{lfm}$, erschlossen, Findbuch)

Inhalt: Korrespondenz mit Fachkollegen, wissenschaftlichen, kulturellen und gesellschaftlichen Einrichtungen, mit Verlagen und Redaktionen sowie privater Schriftwechsel, Materialsammlung zu literarischen Epochen, Themen und Autoren, Typoskripte und Druckvorlagen, persönliche Unterlagen, Sonderdrucke. 


\section{Nerius, Dieter $(* 1935)$}

Universitätsarchiv Rostock

Vorlass (1 lfm, nicht erschlossen)

Inhalt: Orthographietagungen 1978-1991, Ausarbeitungen zur Neuregelung der deutschen Orthographie.

Niewöhner, Heinrich (1889-1959)

Archiv der Berlin-Brandenburgischen Akademie der Wissenschaften

Nachlass (1,6 lfm, erschlossen, Findbuch)

Inhalt: Manuskripte und Arbeitsmaterialien über Heinrich den Teichner, Neues Gesamtabenteuer und zur deutschen Literaturgeschichte, gedruckte Zeitschriftenaufsätze.

Richter, Trude (1899-1989)

Archiv der Akademie der Künste Berlin

Teilnachlass $(0,5 \mathrm{lfm}$, erschlossen, eigene Datenbank)

Inhalt: Werkmanuskripte, u. a. Memoiren und literarische Essays, biographische Unterlagen, Briefwechsel, u. a. mit Lilly Becher, Jewgenia Ginsburg und Anna Seghers, Artikel und Texte über Leben und Werk Trude Richters, Sammlung Hans Günther (1899-1938), Zeitungsausschnitte, Materialsammlung, Fotos und Zeichnungen.

\section{Stadtbibliothek Leipzig}

Teilnachlass $(0,9 \mathrm{lfm}$, erschlossen, Findbuch)

Inhalt: Korrespondenz, Manuskripte und Typoskripte, autobiographisches Material, Fotos, Zeichnungen, Rezensionen, Vorlesungsniederschriften, Bücher.

Schlenstedt, Silvia (1931-2011) und Dieter (1932-2012)

Archiv der Akademie der Künste Berlin, Silvia und Dieter Schlenstedt-Archiv Nachlass (28 lfm, teilverzeichnet, Liste)

Inhalt: Manuskripte wissenschaftlicher und essayistischer Arbeiten, Druckbelege, Korrespondenz, umfangreiche, v.a. projektbezogene Materialsammlungen mit Schwerpunkt auf der Literatur der DDR und der Exilforschung, Unterlagen über die Tätigkeit im Zentralinstitut für Literaturgeschichte bei der Akademie der Wissenschaften der DDR und über die Geschichte des P.E.N.-Zentrum (Ost) in den 1990er Jahren, Arbeiten fremder Autoren.

Schuhmann, Klaus $(* 1935)$

Universitätsarchiv Leipzig

Vorlass $(10,2 \mathrm{lfm}$, nicht erschlossen)

Inhalt: $k$.A.

Steiner, Gerhard (1905-1995)

Archiv der Berlin-Brandenburgischen Akademie der Wissenschaften 
Teilnachlass (3,6 lfm, erschlossen, Verzeichnis)

Inhalt: Persönliche Unterlagen, Korrespondenz, Manuskripte sowie Materialsammlungen u. a. zu J. G. Forster und C. W. Frölich.

\section{Staatsarchiv Meiningen}

Teilnachlass (1,3 lfm, erschlossen, eigene Datenbank)

Inhalt: Persönliche Unterlagen, Korrespondenzen mit Institutionen, Verlagen und Vereinen, Korrespondenzen mit Einzelpersonen, Manuskripte, Materialsammlungen, Forschungen zur Freimaurerei.

\section{Träger, Claus (1927-2005)}

\section{Deutsches Literaturarchiv Marbach}

Nachlass (6 lfm, vorgeordnet, eigene Liste)

Inhalt: Prosa: Manuskripte und Typoskripte zu Reden, Vorträgen, Aufsätzen und Monographien zur Literaturgeschichte und zur Methodologie der Literaturwissenschaft, zu universitären Lehrveranstaltungen, Zeitungs- und Zeitschriftenartikeln, Rezensionen u. a. Verschiedenes: Gutachten, Notizen, Reiseberichte u. a. Briefe an und von: Werner Bahner, Ehrhard Bahr, Ernst Behler, Pierre Bertaux, Elke Blumenthal, Lieselotte Blumenthal, Jurij Brĕzan, Manfred Buhr, Cesare Cases, Walter Dietze, Gisela Dischner-Vogel, Hugo Dyserinck, Martin Fontius, Leonard Wilson Forster, Franz Fühmann, Hans Jürgen Geerdts, Wilhelm Girnus, Peter Hacks, Joachim Hellwig, Jost Hermand, Helmut Holtzhauer, Johannes Janota, Hans Robert Jauß, Werner Krauss, Helmut Kreuzer, Alfred Kurella, Victor Lange, Hans Joachim Mähl, Walter Markov, Hans Marquardt, Hans Mayer, Herman Meyer, Werner Mittenzwei, Manfred Naumann, Matthias Oehme, Helmut Reinalter, Fred Rodrian, Richard Samuel, Hans Jörg Sandkühler, Albrecht Schöne, Herbert Seidler, Walther Siegmund-Schultze, Horst Turk, Robert Weimann, Klaus Weimar, Markus Wolf, Viktor Žmegač, Gerhard Zwerenz u. a., Familienbriefe aus der Kriegsgefangenschaft 1946-1948.

\section{Voegt, Hedwig (1903-1988)}

\section{Universitätsarchiv Leipzig}

Nachlass (2,6 lfm, erschlossen, eigene Datenbank)

Inhalt: Korrespondenz, persönliche Unterlagen zur Biographie, Fotos, Würdigungen, Rezensionen, Manuskripte zu Lehrveranstaltungen und Vorträgen.

\section{Wenzel, Georg $(* 1928)$}

\section{Archiv der Berlin-Brandenburgischen Akademie der Wissenschaften}

Vorlass $(0,3 \mathrm{lfm}$, nicht erschlossen $)$

Inhalt: $\mathrm{k}$.A.

Simone Waidmann, Deutsches Literaturarchiv Marbach, E-Mail: simone.waidmann@dla-marbach.de; Frederike Teweleit, ebd., E-Mail: forschung@dla-marbach.de; Ruth Doersing, ebd., E-Mail: ruth.doersing@dla-marbach.de 